\title{
Des Lumières au Romantisme. Le topos de l'existence en confusion
}

\section{Christian Angelet}

\section{(2) OpenEdition \\ 1 Journals}

Édition électronique

URL : http://journals.openedition.org/rief/202

DOI : 10.4000/rief.202

ISSN : 2240-7456

Éditeur

Seminario di filologia francese

Référence électronique

Christian Angelet, «Des Lumières au Romantisme. Le topos de l'existence en confusion », Revue italienne d'études françaises [En ligne], 3 | 2013, mis en ligne le 15 décembre 2013, consulté le 10 décembre 2020. URL : http://journals.openedition.org/rief/202 ; DOI : https://doi.org/10.4000/rief.202

Ce document a été généré automatiquement le 10 décembre 2020.

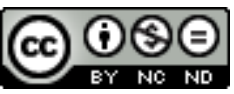

Les contenus de la RIEF sont mis à disposition selon les termes de la Licence Creative Commons Attribution - Pas d'Utilisation Commerciale - Pas de Modification 4.0 International. 


\title{
Des Lumières au Romantisme. Le topos de l'existence en confusion
}

\author{
Christian Angelet
}

1 Comment ressaisir de manière générale le passage de l'idéologie des Lumières au culte romantique de la subjectivité? À l'exaltation du moi vécu comme unique et irremplaçable, tel que l'a affiché Rousseau dans le Prologue des Confessions : "Je ne suis fait comme aucun de ceux que j'ai vus ; j'ose croire n'être fait comme aucun de ceux qui existent. Si je ne vaux pas mieux, au moins, je suis autre $»^{1}$. Des Lumières au Romantisme : rupture ou continuité ? Les deux, sans doute.

2 Pour ponctuer les deux étapes dans toute leur généralité, il me paraît qu'il convient de partir d'une certaine vision de l'homme. La réflexion philosophique du XVIII ${ }^{\mathrm{e}}$ siècle prend couramment son départ dans la fiction de l'homme dit naturel, antérieur à la civilisation et à la vie sociale. Le texte canonique en la matière, on le rencontre dans l'Histoire naturelle de l'Homme de Buffon (1749). C'est l'histoire du premier homme qui s'éveille à la vie. Au début, il est tout entier hors de soi, il n'a pas encore d'identité, pas de moi. Il n'est encore personne. Il ne deviendra un individu que par l'éducation des sens. Pour les contemporains de Buffon, le problème était de poids. Comme on sait, les philosophes sensualistes étaient convaincus de l'influence de la conformation physique de l'homme sur sa métaphysique et sa morale. La plupart de ces auteurs discutent sur la prédominance du toucher et de la vue. Buffon, lui, est convaincu de la priorité du toucher. C'est le toucher qui va enseigner à l'homme qu'il y a un monde en dehors de lui. Mais auparavant, il était un avec la nature, il se confondait avec elle. Cet état primitif, Buffon l'appelle l'existence en confusion. Je ne m'en vais évidemment pas vous lire tout ce texte, seulement la fin. Pour exposer sa thèse, Buffon invente une prosopopée de l'homme primitif :

Je me souviens de cet instant plein de joie et de trouble, où je sentis pour la première fois ma singulière existence; je ne savois ce que j'étois, où j'étois, d'où je venois. J'ouvris les yeux, quel surcroît de sensations ! la lumière, la voûte céleste, la verdure de la terre, le cristal des eaux, tout m'occupoit, m'animoit et me donnoit un sentiment inexprimable de plaisir: je crus d'abord que tous ces objets étoient en moi et faisoient partie de moi-même. ${ }^{2}$ 
Dans cette fiction du premier homme s'éveillant à la connaissance, l'être se perd dans la totalité du spectacle qui l'entoure, il est arrêté sur la sensation visuelle pure, avant la manifestation de l'intelligence, avant sa prise de conscience d'une existence individuelle. Il ne pourra devenir un être particulier et se soustraire à la confusion, à l'effusion de soi dans la nature, que par l'éducation des sens. Dans la suite du texte de Buffon, c'est le toucher qui va instituer et circonscrire son moi.

4 Le passage des Lumières au Romantisme peut s'éclairer à l'aide de ce topos. L'expansion du sujet dans le paysage, la fameuse rêverie identifiante, on sait que les romantiques l'ont intégrée à leur propre vécu. L'unité mystérieuse de l'homme et du monde, n'est-ce pas une formule de base du Romantisme telle que nous l'ont enseignée les manuels et histoires de la littérature?

5 Et puis, les romantiques ont inventé le journal intime. Le premier responsable en la matière serait Maine de Biran. Un nom qui ne dit plus grand-chose, sauf aux spécialistes. Il est répertorié dans les histoires de la philosophie: on lui consacre un copieux commentaire dans la foulée des sensualistes, justement ${ }^{3}$. Mais il figure aussi dans les histoires de la littérature, parmi les premiers romantiques. A juste titre, sans doute. Romantique il l'est, parce qu'il serait l'inventeur du type d'écriture solitaire et introspective qui fera florès après lui. Il va y avoir Senancour, Maurice de Guérin, Benjamin Constant, Frédéric Amiel, Stendhal, Delacroix et tant d'autres. Et l'on sait que c'était un genre promis à un brillant avenir en littérature française.

6 Maine de Biran a vécu de 1766 à 1834. Il a connu la Révolution française et la Restauration. C'était un malchanceux, un déçu, un triste. La grande aventure de sa vie, la seule, cela a été son mariage. Avec sa cousine, une créole dont le mari avait émigré pendant la Révolution. Comme on était resté sans nouvelles de cet homme pendant plusieurs années et qu'il passait pour défunt, Maine de Biran avait épousé la veuve. Mais voilà, après sept ans de bonheur et trois enfants, le mari était réapparu. Le saisissement a été tel que la dame en est tombée malade et a passé outre. Voilà pour la biographie de Maine de Biran. J'ajoute qu'il a été député à partir de 1797 et sous-préfet de l'arrondissement de Bergerac jusqu'à la fin de ses jours. Il s'est beaucoup démené et dévoué pour la cause publique, mais il ne l'appréciait pas. Il n'aura vécu que pour écrire.

7 Je reviens à mon sujet, à savoir l'existence en confusion. D'abord, quelques précisions sur Biran philosophe.

8 Ce n'est évidemment pas le lieu d'exposer son système. Disons que, pour l'essentiel, il porte sur le rapport du physique et du moral et sur la composition du moi. Biran a commencé comme sensualiste et disciple de Buffon. Il cite d'ailleurs in extenso l'Histoire naturelle de l'Homme dont je vous ai lu un extrait en commençant. Le processus est le même chez Buffon et Biran philosophe. Il s'agit de décomposer la pensée pour en retrouver les formes simples et dites primitives. C'est en remontant aux sensations que l'on retrouve ce que l'auteur appelle la simplicité primitive. Ou encore la vérité primitive immédiate. C'est, tout comme chez Buffon, la vérité antérieure à la conscience de l'individualité. Et c'est encore et toujours l'existence en confusion. Biran la définit comme suit : «L'homme ne se distingue pas de prime abord de l'objet de ses représentations. Il existe tout entier hors de lui. La nature est lui, lui est la nature $»^{4}$.

9 Dans cet état purement sensitif, l'homme n'a pas encore de conscience propre. Il est encore étranger à lui-même. Toutefois, à la différence de Buffon et autres sensualistes qui ont marqué ses débuts, notre penseur déclarera en conclusion que l'homme n'a pu 
accéder à son individualité fondamentale que par la volonté. La devise personnelle de Biran était : « volo, ergo sum ».

Voilà pour Biran philosophe des Lumières. De ce dernier à l'écrivain romantique qu'il fut également, il y a un abîme. J'ai dit plus haut qu'il passe pour l'inventeur du journal intime. Qu'est-ce que le journal intime ? Ce n'est pas un genre littéraire, du moins pas à l'origine. Il n'entrera dans le champ des Belles Lettres qu'après coup. Ce n'est même pas un appel à la communication, un échange virtuel, l'espoir d'un entretien futur. Non : on n'écrit que pour soi, et son journal, on le met à l'abri des regards. Autant de cahiers qu'on ne découvrira que post mortem 5 .

Cette pratique de l'écriture en miroir est un indice très net de l'inversion que l'Idéologie des Lumières a subie au siècle suivant. La philosophie était un discours public, une parole extériorisée, un message transmis à tous. Il importait de propager les vérités fondamentales de l'espèce humaine. En définitive, chez Biran philosophe, apôtre de la volonté, il y allait d'une morale. À cela va s'opposer un individualisme forcené, la clôture du moi, le tête-à-tête interminable avec soi-même. Notre auteur est devenu un être avalé par l'écriture et comme résorbé en elle.

Des Lumières au Romantisme : j'ai dit, en commençant, qu'il y aurait à la fois rupture et continuité.

Effectivement, il se trouve que, du premier au dernier d'entre eux, nos intimistes s'empressent de récupérer le topos de l'existence en confusion. Renfermés en eux-mêmes, coupés du monde, ils apparaissent hantés par le rêve de disparaître dans la totalité de la nature, de faire corps et esprit avec elle. L'irréparable clôture du moi se défait en son contraire. Voici, chez Biran, promeneur solitaire, une des premières occurrences du fameux panthéisme mystique qui traversera le XIX ${ }^{\mathrm{e}}$ siècle et prospérera bien au-delà. C'est dans son Journal en date du 17 mai 1815 :

Je promenais lentement, dans une allée de jeunes platanes, que j'ai plantés il y a peu d'années. Sur toutes les impressions et les images vagues, infinies, qui naissaient de la présence des objets et de mes dispositions, planait ce sentiment de l'infini qui nous emporte quelquefois vers ce monde des réalités qui va se rattacher à Dieu, comme à la première et la seule des réalités. ${ }^{6}$

14 En fin de compte, c'est le sensualisme originel du philosophe Biran qui va fournir la matière première et l'outil de son spiritualisme. Toutes les sensations manifestent une vérité religieuse :

Il semble que dans cet état, où toutes les sensations extérieures et intérieures sont calmes et heureuses, il y ait un sens particulier approprié aux choses célestes et qui, enveloppé dans le monde actuel de notre existence, est destiné peut-être à se développer un jour, quand l'âme aura quitté son enveloppe mortelle. ${ }^{7}$

Sensualisme, Romantisme: le temps n'est plus où l'on opposait le Romantisme aux Lumières. L'histoire de la littérature n'est pas faite d'un mouvement progressif et de périodes successives, mais de courants superposés et qui avancent ou rétrogradent.

16 Cela dit, s'agissant de notre topos, où en sommes-nous? Nous sommes passés de l'exposé rationaliste et impersonnel de Buffon au soliloque autobiographique. Un Maine de Biran convoque les deux registres ensemble: c'est un analyste laborieux doublé d'un rêveur impénitent.

17 La communion avec la nature, on sait que les Romantiques vont l'instituer en lieu commun et le moduler à leur guise. Tel est le paradoxe des intimistes : l'écriture en miroir et la hantise de la solitude les portent à la désidentification et à la pluralisation du moi. Ce 
sera un thème récurrent de la rêverie romantique. Il a été abondamment commenté. Toutefois, la critique n'en a guère relevé la préhistoire sensualiste et philosophique ${ }^{8}$.

Alors, pour finir, un exemple parmi d'autres, et non des moindres. Je vais quitter les premiers romantiques pour vous donner lecture et commentaire d'un des plus beaux spécimens de l'existence en confusion. il s'agit d'un poème en prose de Baudelaire intitulé Le Confiteor de l'artiste. C'est la troisième pièce du Spleen de Paris. On sait que Baudelaire représente à la fois un héritier du Romantisme et un puissant rénovateur. Nous sommes en 1862. Il s'agit vraisemblablement d'un texte écrit à Honfleur, à l'embouchure de la Seine et au bord de la mer, où la mère de Baudelaire possédait un petit pavillon. Je vais vous en lire le début. Vous allez reconnaître quelques repères essentiels de la tradition que j'ai évoquée :

Que les fins de journées d'automne sont pénétrantes! Ah! pénétrantes jusqu'à la douleur ! car il est de certaines sensations délicieuses dont le vague n'exclut pas l'intensité ; et il n'est pas de pointe plus acérée que celle de l'Infini.

Grand délice que celui de noyer son regard dans l'immensité du ciel et de la mer! Solitude, silence, incomparable chasteté de l'azur ! une petite voile frissonnante à l'horizon, et qui par sa petitesse et son isolement imite mon irrémédiable existence, mélodie monotone de la houle, toutes ces choses pensent par moi, ou je pense par elles, (car dans la grandeur de la rêverie, le moi se perd vite !) ; elles pensent, dis-je, mais musicalement et pittoresquement, sans arguties, sans syllogismes, sans déductions. ${ }^{9}$

On ne peut nier l'évidence: ce spécimen de contemplation identifiante fait écho à la tradition sensualiste que j'ai évoquée précédemment. On y retrouve les mots-thèmes que j'ai relevés plus haut: les sensations (ici, la vue et l'ouïe), la rêverie qui mène au sentiment de l'infini... Et puis, la métamorphose du moi se produit sur le mode de l'intuition: c'est un retrait en deçà de la raison raisonnante. Cette abolition du moi conscient n'apparaît-elle pas comme la récupération de notre prétendue vérité primitive?

Cet admirable morceau est une formulation de l'art poétique de Baudelaire. En définitive, que veut dire s'incorporer aux choses? Pour un poète, cette opération magique ne peut évidemment avoir lieu que par le truchement de la langue. Être la nature, c'est habiter la langue devenue un corps sonore. Tel est le vrai désir du poète. Et notre bonheur, à nous lecteurs, n'est-il pas d'y demeurer avec lui?

\section{NOTES}

1. J.-J. Rousseau, Confessions, dans Id., CEuvres complètes, éd. sous la direction de B. Gagnebin et M. Raymond, Paris, Gallimard, « Bibliothèque de la Pléiade », 1961-1995, t. I, livre I, p. 5.

2. G.-L. Buffon, Histoire naturelle de l'homme, dans Euvres philosophiques de Buffon, texte établi et présenté par J. Piveteau, Paris, PUF, 1954, p. 309-310.

3. Cf. E. Bréhier, Histoire de la philosophie, Paris, Librairie Félix Alcan, 1938, t. 2, p. 614-646.

4. E. Bréhier, op.cit., p. 625.

5. Voir Maine de Biran, Journal, édition intégrale publiée par H. Gouhier, Neuchâtel, Éditions de la Baconnière, 1954-1957, 3 vol. 
6. Ibid., t. 1, p. 81.

7. Ibidem.

8. Voir M. Raymond, Romantisme et Rêverie, Paris, José Corti, 1978 et C. Angelet, "Paysages philosophiques et rhétoriques ", dans Le Paysage et ses grilles, Colloque de Cerisy, textes réunis et présentés par F. Chenet, Paris, L'Harmattan, 1996, p. 89-99.

9. Ch. Baudelaire, Le Spleen de Paris, dans Euvres complètes, texte établi, présenté et annoté par C. Pichois, Paris, Gallimard, « Bibliothèque de la Pléiade », 1975-1976, 2 vol. , t. I, p. 278.

INDEX

Mots-clés : Lumières, romantisme, sensualisme, Buffon (Georges-Louis Leclerc comte de), spiritualisme, Maine de Biran, Baudelaire (Charles) 